\title{
Foul or no Foul? Effects of Permitted Fouls on the Defence Performance in Team Handball
}

\author{
by \\ Frowin Fasold ${ }^{1}$, Dennis Redlich ${ }^{1}$
}

\begin{abstract}
Attacking phases in team handball are highly dynamic, characterized by fast movements and a high frequency of fast passes with the aim to score a goal. Consequently, the opposing/defending team tries to prevent successful offensive actions by restraining the offensive opportunities and the probabilities of scoring a goal. According to the rules, defensive players are allowed to use body contact (e.g. with bent arms) to get in and keep their optimal defending position. If such a contact is not in line with the rules (e.g. clutching or pushing) and results in a turnover of ball possession, a foul is called and a free-throw awarded. However, there is a lack of research answering the question if a permitted foul (without personal sanction) is an effective way to increase the probability of defending the own goal successfully, because afterwards the attacking team keeps possession of the ball. Thus, we investigated 1052 attacking phases during games at the elite level to verify whether fouls committed by a defender influenced successful attacking (goal vs. no goal). It was found that when the attack was interrupted by a permitted foul, $50.60 \%$ of the attacks ended with a goal. Yet, when no foul was committed, only $47.09 \%$ of the attacks ended with a goal, however, the analysis revealed that this difference was not significant. Therefore, we concluded that neither a strategy of stopping offensive actions by body contact nor avoiding fouls and focusing only on intercepting the ball were favourable solutions in successful defending in team handball. It seems effective to implement a defence strategy with clearly defined fouling zones and situations, to deal with the tactical possibility of permitted fouls in handball. This idea and further considerations are discussed for sports practice.
\end{abstract}

Key words: team, team sports, game analysis, tactics, game interruption.

\section{Introduction}

The game idea of team handball consists of attacking the other goal while trying to recover possession of the ball by defending the own goal (Griffin and Butler, 2005). The game is characterized by fast game pace, a high number of one-on-one actions (Wagner et al., 2014) and therefore, a high number of possible fouls committed. Until now different variables (focussing on offence, defence and goalkeeper perspectives) have been considered as performance indicators (Bilge, 2012; Daza et al., 2017). Despite the high frequency of fouls committed, the effect of fouls on performance has not been analysed so far, remains unclear and needs further investigation.
The game structure of team handball can be separated into three relevant phases: the attack (in possession of the ball, trying to score), the defence and the goalkeeping (both trying to prevent scoring of the opponent) (Daza et al., 2017). Counterattacks after winning the ball are considered as a sub-phase of the attack phase, whereas the fast back-up after losing the ball is defined as sub-phase of the defence phase (as well as goalkeeping) (Karcher and Buchheit, 2014).

Compared to other sports games (e.g basketball, field hockey), the option of committing a foul sanctioned by a free-throw plays a special role in team handball. For defenders, it is allowed to have body contact (e.g. use of upper arms, bent

\footnotetext{
1 - Institute of Exercise Training and Sports Informatics, Department Cognitive and Team/Racket Sport Research, German Sport University Cologne, Cologne, Germany.
} 
arms, the own trunk; International Handball Federation, 2016) with the opposing attackers. Thus, these kinds of body contact can be used to keep an optimal defending position and prevent the opponent from scoring without receiving a personal punishment. If such body contact is not in line with the rules, but provokes a turnover or technical rule mistake by the attacking opponent, this action will be called as a foul and sanctioned by a free-throw or a penalty and if necessary, underpinned by a personal sanction $(2 \mathrm{~min}$ suspension, yellow or red card). In the current paper we label these kinds of fouls as permitted fouls.

Since in free-throw situations defenders can always get in a positon between the attacker (in possession of the ball) and the own goal (in compliance with a distance of $3 \mathrm{~m}$; International Handball Federation, 2016), a free-throw cannot be seen as a situation favouring the team in the attack. Therefore, permitted fouls through body contact by the defence in team handball are considered a promising strategy to influence the opponents' game flow and dynamics and increase the defence success rate (no goal). Then again, if a free-throw is called, the defence fails to get in possession of the ball (and to start a counterattack). Therefore, it could also be assumed that winning the ball without a foul is a more promising strategy for the defending team.

To contrast the effectivity of both approaches (committing permitted fouls or not) in team handball, we conducted a study to investigate the relationship between permitted fouls and successful defending (as measured by the number of no goal situations for the attack).

Research shows that elite team handball players have about 37 body contact situations (low-intensity-, high-intensity-contact, clasping) per game (Michalsik et al., 2015) and up to 19 direct defence duels (Póvoas et al., 2012). From a theoretical perspective, all these duels and contact situations could lead to an interruption of the game (referee call). Research shows a relatively stable number of about 57.55 attacking phases (ball possession situations) per team during a game (Bilge, 2012). Approximately, half of these attacks $(52.8 \%)$ are interrupted (Karcher and Buchheit, 2014). Unfortunately, the reasons of these interruptions (e.g. fouls, time-outs, outs) are unclear. Though, there is no data about the frequencies of permitted fouls per game called by the referees.

In consideration of those points in practice, Brack (2002) developed a gameeffectiveness-index based on frequencies and weightings of defined game actions, which indicates inter alia a possible effect of fouls committed on individual game performance. In this concept the action of a permitted foul as a game interruption is weighted with a positive value $(+0.2$; compared to the highest possible weighting of scoring goal with +1.0 ). It seems to be important that tactical variables (e.g. time, space, situation) are integrated in such a rating, but unfortunately, the reasons of the weighting of fouls are not precisely stated by Brack (2002). Without keeping such variables in mind, the active use of permitted fouls is seen as a positive factor for the success in team handball by Brack (2002).

With the objective to design an easy-touse match statistics sheet, Marczinka (2013) integrated a "positive foul" action in the evaluation part of defence activities. Based on the statements "...the player in defence stops the attacker with the ball by checking and this is a positive remark..." and "...the team that has more marks in the section of 'positive fouls' (stopping the attacker with ball by checking) wins the game..." (Marczinka, 2013), it may be assumed that game interruptions by committing permitted fouls are positive in consideration of game success.

Furthermore, the longer an attacking phase lasts, the higher is the risk that referees forewarn the attacking team for passive play. The higher probability of forewarning signals for passive play increases the pressure on the attacking team and should decrease the frequency of successful scoring attempts. Therefore, permitted fouls could extend the duration of an attacking phase and increase the success of the defending team (avoiding a goal).

In contrast to the previously mentioned findings and from a more practical point of view, statements of successful coaches such as Heiner Brand and Claude Onesta, do not confirm the effectiveness of committing fouls in general. According to Brand (2008), the defence should not only aim to destruct offensive movements; the aim of defending should rather be winning the 
ball. Similarly, Onesta has stated that committing fouls is only useful when control over the offence action gets lost and a foul is necessary to rebuild an effective and stable defence. Interruptions of the opponents' attack, by committing a foul without good reasons, should be avoided (Landuré, 2011).

In conclusion, knowledge and behaviours dealing with permitted fouls in handball are mostly based on game-specific behaviours and experiences and there is a lack of empirical validations of these behaviours which could result in recommendations for sports practice (using keywords like a foul and handball in scientific databases, e.g. web of science, PubMed, reveals only sources related to sports medicine or referee decision making). Therefore, it remains unknown whether fouling is effective for success in handball or not.

The small body of literature leads to various findings and theories about the effect of (permitted) fouls in handball. Consequently, it is hard to derive clear hypotheses with regard to the relationship between frequencies of fouls committed and successful defending. Therefore, the purpose of the present study was to investigate the effect of permitted fouls (followed by free-throws without personal sanctions) on the success of a defence (no goal by the opponent attack) within single events. Additionally, we evaluated how many permitted fouls were committed per game and if the number of successful defence actions was influenced by the number of permitted fouls. Furthermore, we investigated whether the number of committed fouls influenced the frequency of forewarning signals for passive play and whether the effect of fouls on the opposing scoring probability persisted, also after the forewarning signal for passive play.

\section{Methods}

To answer our research questions, we examined video footage of handball games in a post-hoc observation study.

\section{Material}

We chose 88 videotaped games of male team handball from a single season (2015/2016) of the $1^{\text {st }}$, the $2^{\text {nd }}$ and four $3^{\text {rd }}$ leagues (north, east, west, south) in Germany. With the aim to include at least 1000 actions, we randomly chose ten games out of the matches played in four $3^{\text {rd }}$ leagues, 25 games out of matches played in the $2^{\text {nd }}$ league and 25 games from the matches played in the $1^{\text {st }}$ league. Because of technical inadequacies of the available video footage, only 24 games of the $1^{\text {st }}$ league and 21 games of the $2^{\text {nd }}$ league could be analysed. To include a high number of different teams in our analyses, every team was only allowed to participate in a maximum of two games.

\section{Design and Procedures}

To avoid game-effects occurring at the beginning or the end of a match such as testing the limits of the referees (e.g. permitted hardness in body contacts), fatigue effects, personal duels or crunch-time-factors (e.g. special team tactics like one-on-one defence), we only analysed the actions between the $10^{\text {th }}$ and $20^{\text {th }}$ minute of each game. Only the attacks against organized defences in 6 vs. 6 situations were considered. Furthermore, only such situations were taken into consideration in which all defending players were positioned between the middle-line and the defending goal, when the ball crossed the middle line and the attacking team apparently did not force the game speed at that moment. Thus, all the other game situations (e.g. 6 vs. 5,5 vs. 6,5 vs. 5 ) and all counter attacks/back-up phases were not considered. In the event of personal sanctions, an action remained in the analysis if the referees showed a defender a yellow card. When the referees sanctioned a defender with a $2 \mathrm{~min}$ suspension or a red card and awarded a $7 \mathrm{~m}$ penalty, the action also remained in the analysis. When a suspension was awarded, but not penalized by a $7 \mathrm{~m}$-penalty, the action was excluded.

The remaining scenes were analysed with regard to the objective frequency variables: foul (yes, no), cards (yellow, red), time-suspension followed by a penalty, forewarning signal for passive play, the attack outcome (goal, technical mistake, missed shot) and the defence outcome (blocks, interceptions). The offence and defence outcomes were categorized into goal and no goal actions. We analysed the effect of the factors foul (foul vs. no foul), and the number of fouls (once vs. twice vs. ...x) on the dependent variables forewarning signal for passive play (passive vs. no passive) and goal (goal vs. no goal). To clarify the relationship between factors foul and forewarning 
signal for passive play, we continued to investigate the effect of these factors on the dependent variable goal in the second analysis.

Evaluation of the video footage and the preparation of the data were conducted by an experienced handball player and coach $(>10$ years playing experience in the $2^{\text {nd }}$ league, -license coach German Handball Federation). Although, the definitions in the evaluation of the actions where clear and mainly linked with objective criteria (referee calls), we performed occasionally a reliability test. The second judge $(>12$ years playing experience, -license) evaluated ten randomly chosen games of the analysed sample. With a Cronbach's $\alpha>.92$ for all observed variables, the reliability for the study was high.

\section{Statistical Analysis}

We used $2 \times 2$ tables for testing the general distributions of the single variables and the effects of frequencies of the independent factors on the frequencies of the dependent variable. To avoid rather highly conservative results we conducted a Barnard's unconditional test (Mehrotra et al., 2003) for the frequency comparisons. Concerning the effect sizes, Cramer's $\varphi$ was reported.

\section{Results}

The final analysis consisted of 1127 attacking actions. A necessary reduction (e.g. unclear situations according to our definitions) led to a total of 1052 attacking actions included in the statistical analysis. In $39.64 \%$ of the observed actions, the defending team interrupted the attack by committing a foul (referee call). In $11.79 \%$ of the situations, the referees sanctioned the foul with a yellow card, in $1.62 \%$ with a $2 \mathrm{~min}$ suspension (which was followed by a $7 \mathrm{~m}$ penalty). Excluding the situations in which a player received a personal sanction, and forecasting the frequencies of fouls on $60 \mathrm{~min}$ playing time, we registered about 21 fouls per game.

In a first step, we calculated a base rate for the variables goal vs. no goal by using the absolute distribution of successful and non-successful attacks in our sample (Table 1). This distribution does not significantly differ from an equal distribution, $p=.52, \varphi=0.02$, and is quite similar to results of previous studies (50.86 success rate in the attack phase; Bilge, 2012). We then used this base rate as expected frequencies for the event goal for further steps of the data analysis.

The frequency distribution of the goal vs. no goal situation, in all actions in which the defence interrupted offensive actions with a foul was similar to the base rate, $p=.52, \varphi=0.01$. In actions without a foul, the frequency of the goal situation decreased, but did not significantly differ from the base rate, $p=.59, \varphi=0.02$.

Within the foul actions, the defence interrupted the attack once in $65.47 \%$, twice in $25.90 \%$, three times in $7.43 \%$ and four times in $1.20 \%$ of all the cases. Due to a small amount of cases with three or more fouls, we summarized those cases to avoid losing them for the statistical analysis. Neither the one foul situations $(n=273$, $51.28 \%$ goals vs. $48.72 \%$ no goals, $p=.52, \varphi=0.02$ ), nor the two foul situations $(n=108,51.85 \%$ goals vs. $48.15 \%$ no goals, $p=.53, \varphi=0.01$ ) significantly differed from the base rate of goal and no goal situations. In the three and four foul situations the frequency of goal decreased $(n=36,41.67 \%$ goals vs. $58.33 \%$ no goals, $p=.51, \varphi=0.02)$, but this distribution was also not significantly different from the expected base rates.

Only in $6.65 \%$ of the 1052 situations, the referees decided to forewarn the attacking team for passive play (please note that the games were videotaped before the rule changes for passive play in 2016). In situations with a committed foul, the number of passive play situations was significantly higher than without a foul (foul $n=$ $10.00 \%$ vs. no foul $n=90.00 \%, p<.01, \varphi=0.27$ ). The frequency of the event goal decreased in situations with a foul and with passive play, but the frequency distribution did not significantly differ from the base rate, $p=.11, \varphi=0.05$ (Figure 2). In situations with a defensive foul without passive play, the frequency distribution was equal to the base rate, $p=.16, \varphi=0.04$ (Figure 2). The descriptive visible effects (Figure 2) of the situations without a foul and passive play, $p=.32$, $\varphi=0.03$, as well as without a foul and without passive play, $p=.71, \varphi=0.01$, did not reach the level of significance compared with the base rate. 

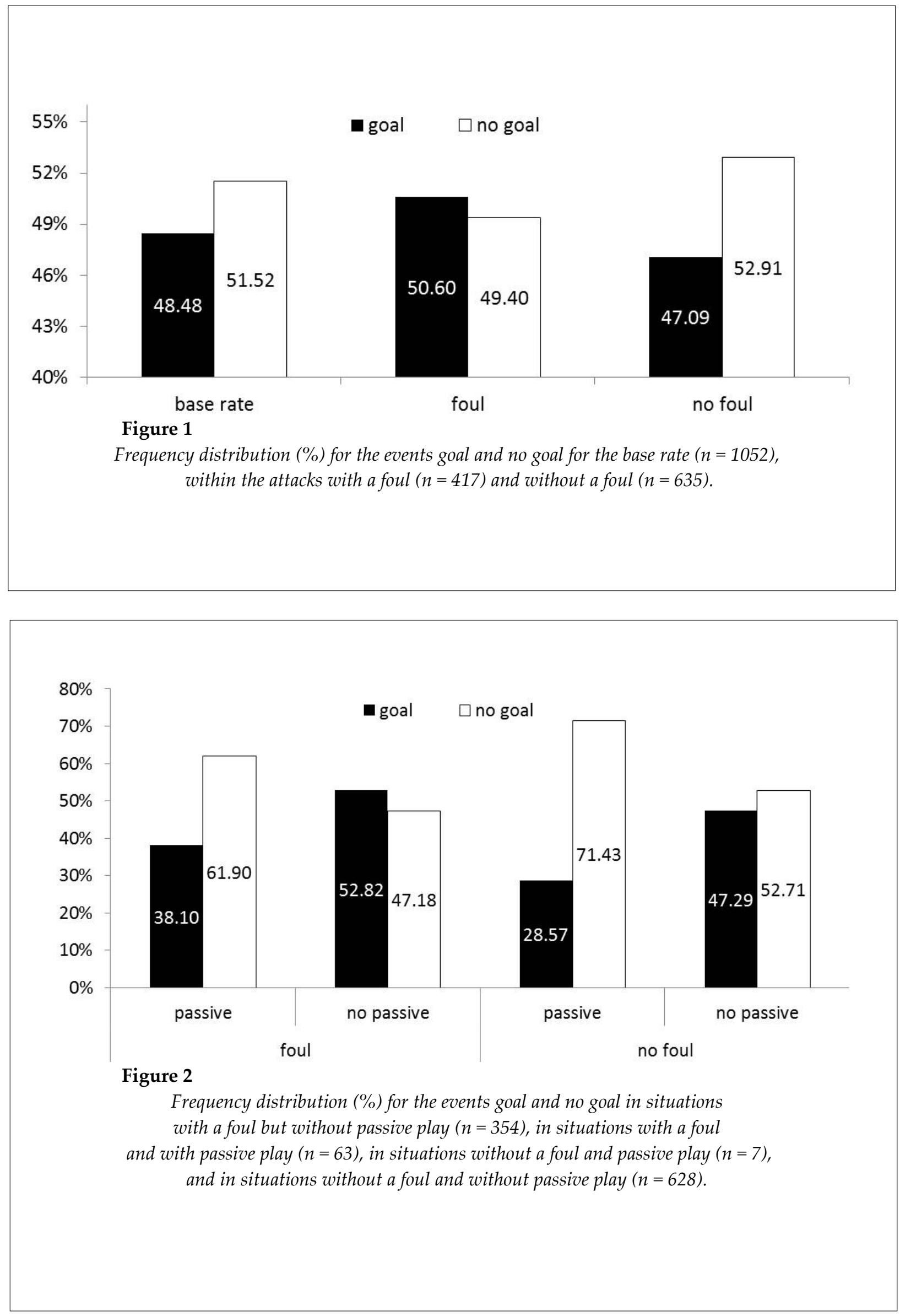

(C) Editorial Committee of Journal of Human Kinetics 


\section{Discussion}

Whereas the number of personal sanctions is extensively documented for elite level handball (e.g. 2012/13 there was an average of 4.2 2 min-suspensions per game in the first Spanish Handball League; Gómez et al., 2014), there is a lack of knowledge about the number of permitted fouls within a game and additionally whether permitted fouls influence the success of defending. Our data showed that about 21 permitted fouls were committed per game during the regular attack phases, which is, on a first sight surprisingly low from a practitioner's perspective. This is more the case if you consider that according to Karcher and Buchheit (2014) the attacking phase captures $88.2 \%$ of the game time. An explanation might be that we chose to analyse only regular attacking situations and therefore, excluded counter attack- and back-up phases as well as situations after time suspension (e.g. 5 v. 6). Additionally, there are further unsanctioned game interruptions, like when a defender touches the ball unintentionally with the feet, or steps in the own goal-area while taking ball possession. In line with data from Karcher and Buchheit (2014), we assumed that half of the attack phases per game was interrupted, but approximately $40 \%$ of those interruptions were regarded as permitted fouls, due to the definition assumed in this paper.

Our results show a small negative effect of committing fouls on the success of defending (Figure 1). In detail, the defence success rate (no goal) increased by $3.15 \%$ if no foul was committed. However, this effect is far from statistical significance (compared to the base rate). Contrarily, when the defence committed more than three fouls per defence phase, the success rate increased by $9.62 \%$. This tendency does not seem to reach significance, probably because of the small number of actions (36 cases of three and four fouls).

Interestingly, our results show that permitted fouls were positively related with the forewarning for passive play. Thus, we assume that fouls extend the duration of the attacking phase, so that through this extension referees are more inclined to show a forewarning signal. The effect of passive play is intriguing considering that the frequencies of successful defence actions increase (with a foul to $10.38 \%$, without a foul to $19.91 \%$ ) compared to the base rate. One limitation of this interpretation is the small sample size, and probably as a consequence the non-significant statistical results. That is why our interpretation is based on descriptive results, but lacks statistical power.

Based on our results, neither interrupting the attack of the opponent as often as possible, nor playing defence without committing a foul, can be seen as a favourable tactical defence strategy. However, our results are in contrast to the statement of Marczinka (2011) that teams with a higher frequency of permitted fouls are more successful, keeping this limitation in mind that we investigated only single events/game actions and not the final game result. While the definitions of foul situations in Brack (2008) are slightly unclear, our data support a neutral weighting of the factor foul in a model predicting team success. Thus, the positive weighting of fouls in the gameeffectivity-index of Brack (2008) should be interpreted with caution. Furthermore, our results are in line with the statement of Claude Onesta (Landuré, 2011) who prefers a tactical approach in which fouls are only considered useful if this is the only option to prevent the attacking opponent from a good scoring position. Consequently, winning the ball should be the first aim of defending (Brand, 2008) and if this is not possible (anymore), permitted fouls should be the necessary tactical solution.

Based on our data and the mentioned statements, we suggest defence tactics with foul zones (zones in which offensive players score with a high probability), in which permitted fouls are necessary, and non-foul zones (offensive players can only score with a low probability), in which fouls should be avoided. These zones could be adapted based on prerequisites and possibilities of the own defence, as well the opponents' attack. Furthermore, decisions to commit a foul or not could be based on the following variables: movement speed of the attacker, position of the attacker in relation to the goal, if the attacking player is allowed to dribble the ball and how his technical/tactical skills are developed. Therefore, additionally to the foul/non-foul zones, definitions of foul and nonfoul situations must be integrated into the defence tactics.

Considering the conducted study, the limitation that we did not investigate a correlation 
between permitted fouls and game success should be kept in mind. With the reduction of the data on the neutral game time (10th to 20th minute), we only investigated a general relationship of the tactical tool, i.e. permitted fouls, on the success of the defence in single play. We have to yet consider that the data could change in different game situations (e.g. the last five minutes).

In conclusion, our study observing permitted foul situations at the elite handball level, showed no effect of the frequency of fouls committed on the success of a defending team (no goal scored by the offense). Nevertheless, permitted fouls seem to be a possible option, especially if the defence has already lost control over the opponents' attack. To evaluate the topic of permitted fouls in future, we suggest further studies including, as mentioned above, more aspects of the analysed situations, like the suggested fouling zones, game time courses or personal/expertise oriented variables. Furthermore, the effect of fouls with punishments (suspension) as a tactical tool could be in focus of future investigations.

\section{References}

Bilge M. Game Analysis of Olympic, World and European Championships in Men's Handball. J Hum Kinet, 2012; 35: 109-118. doi: 10.2478/v10078-012-0084-7

Brack R. Sports games specific training theory. Scientific and object-theoretical fundaments at the example Handball. Hamburg: Czwalina; 2002

Brand H. Handball. My game, my style. Practising, playing, coaching. Münster: Philippka; 2008

Daza G, Andrés A, Tarragó R. Match statistics as predictors of team's performance in elite competitive handball. RICYDE internacional de ciencas del deporte, 2017; 48: 149-161. doi: 10.5232/ricyde2017.04805

Griffin L, Butler J. Teaching Games for Understanding. Theory, Research, and Practice. Champaign, IL: Human Kinetics; 2005

Gómez MA, Lago-Peñas C, Viaño J, González-Garcia I. Effects of game location, team quality and final outcome on game-related statistics in professional handball close games. Kinesiology, 2014; 46: 249-257

International Handball Federation. Rules of the Game. Indoor Handball. Edition 1. July 2016. Basle; 2016

Karcher C, Buchheit M. On-court demands of elite handball, with special reference to playing positions. Sports Med, 2014; 44: 797-814. doi: 10.1007/s40279-014-0164-z

Landuré P. Prevent the actions of the opponent. The defence-play of the World-Champion. Part 1. handballtraining, 2011; 5+6: 7-17

Marczinka Z. Performance analysis. A way to improve coaching methods in handball. In European Handball Fedaration (eds.), $2^{\text {nd }}$ EHF Scientific Conference. Woman and Handball. Scientific and practical approaches, 2013; 249-255

Mehrotra D, Chan I, Berger R. A cautionary note on exact unconditional inference for a difference between two independent binomial proportions. Biometrics, 2003; 59: 441-450. doi: 10.1111/1541-0420.00051

Michalsik LB, Madsen K, Aagaard P. Technical match characteristics and influence of body anthropometry on playing performance in male elite team handball. J Strength Cond Res, 2015; 29: 416-428. doi: 10.1519/JSC.0000000000000595

Povoas SCA, Seabra AFT, Ascensao A, Magalhaes J, Soares JMC, Rebelo ANC. Physical and physiological demands of elite team handball. J Strength Cond Res, 2012; 26: 3365-3375. doi: 10.1519/JSC.0b013e318248aeee

Wagner H, Finkenzeller T, Würth S, Duvillard SP. Individual and team performance in Team-Handball: A review. J Sport Sci Med, 2014; 13: 808-816

\section{Corresponding author:}

Frowin Fasold

Institute of Exercise Science and Sports Informatics, Department Cognitive and Team/Racket Sport Research, German Sport University Cologne, Müngersdorf 6, 50933 Cologne, Germany

Phone: + 49 (0) 2214982 4293;

E-mail: f.fasold@dshs-koeln.de 